\title{
Projeto lúdico para crianças hospitalizadas: um relato de experiência
}

\author{
Play project for hospitalized children: an experience report
}

Proyecto de juego para niños hospitalizados: un informe de experiencia

\begin{abstract}
Elaine Cristina Rodrigues Gesteira ${ }^{1 *}$, Wiara Viana Ferreira ${ }^{1}$, Stênio Henrique Oliveira ${ }^{1}$, Alice Mendes Machado ${ }^{1}$, Jonathan Estevam dos Santos ${ }^{1}$, Mateus Martins Germano ${ }^{1}$, Rayane Lara Silva ${ }^{1}$, Pedro Júnio Alexandre Santos².
\end{abstract}

\section{RESUMO}

Objetivo: Suscitar uma reflexão para a equipe multiprofissional sobre a importância da atividade lúdica para crianças hospitalizadas. Relato de experiência: O projeto foi conduzido em duas instituições do centro-oeste mineiro, onde os acadêmicos de enfermagem atuavam como contadores de histórias às crianças hospitalizadas, os quais foram capacitados pelo docente orientador e enfermeiros. As histórias eram baseadas nos clássicos infantis, e diferentes enredos associados ao uso de músicas, fantoches e fantasias que favoreciam a imaginação de crianças e acompanhantes, promovendo a humanização e a minimização dos efeitos da doença e da internação para a criança e sua família. Considerações finais: A elaboração e execução desse projeto permitiu aos profissionais de saúde uma reflexão acerca do lúdico na vida de crianças hospitalizadas, como estratégia de cuidado, atenção, comunicação e formação de vínculo. Para os acadêmicos foi uma experiência na prática, em que o processo dialógico entre a Universidade e as instituições de saúde favoreceu a interdisciplinaridade e interprofissionalidade necessárias a formação do aluno, destacando-se em uma aprendizagem significativa de um projeto com relevância social e humanitária.

Palavras-chave: Criança hospitalizada, Humanização da assistência, Jogos e brinquedos.

\begin{abstract}
Objective: To raise a reflection for the multiprofessional team on the importance of playful activities for hospitalized children. Experience report: The project was conducted in two institutions in the Midwest of Minas Gerais, where nursing students acted as storytellers for hospitalized children, who were trained by the guiding professor and nurses. The stories were based on children's classics, and different plots associated with the use of music, puppets and costumes that favored the imagination of children and companions, promoting humanization and minimizing the effects of the disease and hospitalization for the child and his family. Final considerations: The elaboration and execution of this project allowed health professionals to reflect on playfulness in the lives of hospitalized children, as a strategy for care, attention, communication and bonding. For the academics it was an experience in practice, in which the dialogical process between the University and the health institutions favored the interdisciplinarity and interprofessionality necessary for the student's training, standing out in a significant learning of a project with social and humanitarian relevance.
\end{abstract}

Keywords: Child hospitalized, Humanization of assistance, Play and playthings.

\section{RESUMEN}

Objetivo: Suscitar una reflexión para el equipo multiprofesional sobre la importancia de las actividades lúdicas para los niños hospitalizados. Informe de experiencia: El proyecto se llevó a cabo en dos instituciones del Medio Oeste de Minas Gerais, donde estudiantes de enfermería actuaron como narradores de cuentos para niños hospitalizados, quienes fueron capacitados por el profesor guía y enfermeras. Los cuentos se basaron en clásicos infantiles, y diferentes tramas asociadas al uso de música, títeres y disfraces que favorecieron la imaginación de niños y acompañantes, promoviendo la humanización y minimizando los efectos de la enfermedad y la hospitalización para el niño y su familia. Consideraciones finales: La elaboración y ejecución de este proyecto permitió a los profesionales de la salud reflexionar sobre la alegría en la vida de los niños hospitalizados, como estrategia de cuidado, atención, comunicación y vinculación. Para los académicos fue una experiencia en la práctica, en la que el proceso de diálogo entre la Universidad y las instituciones de salud favoreció la interdisciplinariedad e interprofesionalidad necesaria para la formación del estudiante, destacándose en un aprendizaje significativo de un proyecto con relevancia social y humanitaria.

Palabras clave: Niño hospitalizado, Humanización de la atención, Juego e implementos de juego.

1Universidade Federal de São João del Rei (UFSJ), Divinópolis - MG

*E-mail: elaine_gesteira@ufsj.edu.br

${ }^{2}$ Faculdade Pitágoras, Divinópolis - MG

SUBMETIDO EM: 7/2020 


\section{INTRODUÇÃO}

A hospitalização pediátrica é um momento adverso na vida da criança e de sua família, sendo considerada uma situação traumática envolvendo sentimentos de angústia, impotência e sofrimento. A criança enferma sofre com as mudanças e rotinas impostas pela doença, e pelo ambiente hospitalar, o qual se trata de um ambiente desconhecido e ameaçador (GIACOMELLO KJ e MELO LL, 2019; SILVA ACP, et al.,2020).

Além disso, as crianças são submetidas as intervenções que invasivas ou não provocam o medo e a insegurança, suas atividades diárias são interrompidas afastando-as dos amigos, da escola e de seu convívio familiar, sendo o impacto biopsicossocial, inevitável. Porém, se faz necessário que medidas sejam tomadas para amenizar esta experiência, e desse modo, as instituições contam com ferramentas lúdicas e brinquedotecas em seus espaços a fim de estimular o desenvolvimento infantil através do brincar (SILVA DO, et al., 2018).

Sendo assim, na busca pelo cuidado não traumático, vemos a obrigatoriedade da brinquedoteca nos hospitais pela Lei $n$. 11.104 de 21/03/2005 apoiando a criança para que não deixe de brincar somente porque está hospitalizada, pois no brincar a criança se desenvolve, liberando os anseios e apreensões decorrentes da internação (BRASIL,2005).

Nesse sentido, a disciplina Prática de Integração Ensino, Serviço e Comunidade do curso de Enfermagem da Universidade Federal de São João del Rei (UFSJ) proporciona aos acadêmicos do $5^{\circ}$ semestre do curso a experiência no cuidado à criança e ao adolescente com agravo, no âmbito hospitalar. Os conteúdos teóricopráticos salientam sobre a necessidade do ambiente lúdico, e de intervenções de enfermagem que preparem o público infantil para os procedimentos, de forma humanizada e facilitada por brincadeiras, leituras, jogos, músicas e utilização da brinquedoteca, a fim de minimizar os efeitos da hospitalização.

De acordo com o Programa Nacional de Humanização da Atenção e Gestão do Sistema Único de Saúde (SUS) - o HumanizaSUS as instituições de saúde devem proporcionar aos usuários, espaços de humanização (BRASIL, 2013). Frente ao exposto foi observado que as unidades pediátricas que sediam as práticas da referida disciplina possuem brinquedotecas, mas há uma escassez de projetos lúdicos, como a leitura de contos infantis.

Assim foi pensado o projeto "Contos Infantis", a fim de contribuir na política de humanização do SUS durante a hospitalização da criança. Essa ação permite o estímulo ao desenvolvimento infantil, por meio da ludicidade, o que estimula o imaginário infantil. Ademais, esse projeto lúdico é uma ferramenta de sensibilização para toda a equipe multiprofissional que atua na intervenção e recuperação das crianças enfermas. Este relato tem como objetivo suscitar uma reflexão para equipe multiprofissional sobre a importância da atividade lúdica para crianças hospitalizadas.

\section{RELATO DA EXPERIÊNCIA}

Trata-se de um estudo descritivo, na modalidade relato de experiência, sobre a vivência de acadêmicos do curso de enfermagem no projeto de extensão "Contos infantojuvenis para crianças hospitalizadas: estímulo à imaginação" da UFSJ desenvolvidos nos setores pediátricos de um hospital de médio porte e uma unidade de pronto-atendimento (UPA) na região centro-oeste mineira.

As ações ocorreram no período de março de 2019 a fevereiro de 2020, sendo realizadas por cinco acadêmicos do curso de enfermagem, voluntários, coordenados por um docente e dois enfermeiros. Antes de iniciar as atividades, os acadêmicos participaram de capacitações promovidas pelo docente, a fim de se embasarem teoricamente sobre a temática, bem como, prepará-los na atuação de contar histórias para crianças hospitalizadas em diferentes faixas etárias, além de capacitá-los para a elaboração de resumos destinados a eventos científicos nacionais, regionais e locais.

Após a aprovação do projeto pelo edital 11/2018 no Programa de Extensão (PROEX) da UFSJ, procedeuse o contato com as instituições do município. Em ambas as unidades de pediatria os acadêmicos foram apresentados às equipes multiprofissionais, compostas por enfermeiros, técnicos de enfermagem, médicos, residentes, estagiários, nutricionistas, fonoaudiólogas e psicólogos, entre outros profissionais. 
Na ocasião foi apresentado o principal objetivo do projeto, o qual era a minimização dos efeitos da hospitalização pediátrica por meio da mediação de contos infanto-juvenis, bem como, a sua operalização no setor. Nesse contexto, as ações desenvolvidas contavam com o apoio da Pastoral de Humanização e Voluntariado do Hospital e da Gerência de Enfermagem da UPA, as quais permitiam a entrada dos acadêmicos em dias e horários programados e comunicados previamente pela docente coordenadora do projeto.

O público-alvo foi composto por crianças de dois a doze anos de idade e de seus acompanhantes que concordavam em participar da ação extensionista. Desse modo, os acadêmicos contavam as histórias dos clássicos infantis nos quartos das crianças, sendo que as mais debilitadas, ouviam e participavam interagindo em seus leitos. A metodologia de implementação foi desenvolvida em quatro etapas de preparação/capacitação dos extensionistas de acordo com o Quadro 1.

Quadro 1 - Descrição geral das atividades de preparação/capacitação da equipe, 2019.

\begin{tabular}{|c|c|c|}
\hline $\begin{array}{c}\text { Etapas e Carga } \\
\text { horária(CH) }\end{array}$ & Participantes e mediadores & $\begin{array}{c}\text { Tema principal das } \\
\text { atividades }\end{array}$ \\
\hline $1-\mathrm{CH}(8 \mathrm{~h})$ & $\begin{array}{c}\text { Acadêmicos de Enfermagem } \\
\text { Docente do projeto (mediador) }\end{array}$ & $\begin{array}{c}\text { Crescimento e } \\
\text { desenvolvimento infantile }\end{array}$ \\
\hline $2-\mathrm{CH}(4 \mathrm{~h})$ & $\begin{array}{c}\text { Acadêmicos de Enfermagem } \\
\text { Docente do projeto (mediador) } \\
\text { Enfermeiros do projeto }\end{array}$ & $\begin{array}{c}\text { Implicações da } \\
\text { hospitalização infantile }\end{array}$ \\
\hline $3-\mathrm{CH}(4 \mathrm{~h})$ & $\begin{array}{c}\text { Acadêmicos de Enfermagem } \\
\text { Docente do projeto } \\
\text { Enfermeiros do projeto }\end{array}$ & $\begin{array}{c}\text { Como contar histórias } \\
\text { para crianças e } \\
\text { adolescentes }\end{array}$ \\
\hline $4-\mathrm{CH}(4 \mathrm{~h})$ & Profissional pedagogo e contista (mediador) & $\begin{array}{c}\text { Acadêmicos de Enfermagem } \\
\text { Docente do projeto } \\
\text { Enfermeiros do projeto }\end{array}$ \\
& Equipe de enfermagem das instituições participantes & $\begin{array}{c}\text { Visita aos cenários de } \\
\text { prática }\end{array}$ \\
\hline
\end{tabular}

Fonte: Gesteira ECR, et al., 2020.

Conforme a descrição no quadro anterior, as atividades realizadas foram programadas e planejadas previamente, sendo os temas especificados a seguir: Primeira Etapa: foi conduzida uma aula expositiva e dialogada sobre o conceito de crescimento e desenvolvimento na primeira e segunda infância, destacando as alterações físicas, motoras, sensoriais, vocalização, socialização e cognição, linguagem, relações familiares, socialização, personalidade, moralidade e cognição de acordo com Freud, Erikson e Piaget.

Segunda Etapa: foi realizada uma oficina teórico-prática sobre o contexto da unidade pediátrica, os comportamentos da criança frente a hospitalização, a adaptação da criança e de sua família no ambiente pediátrico e a necessidade do brincar, incluindo o ouvir, a interação e a imaginação proporcionadas pelas histórias dos clássicos infantis.

Terceira Etapa: nesta etapa, os acadêmicos e o docente orientador do projeto aprenderam o manejo das leituras, como a escolha do melhor enredo, uso de acessórios, fantoches, cantigas, jogos para interação, tonalidade da voz durante a narrativa, ênfase em momentos das narrativas em que geram suspense, aventura, reflexões, e por fim a avaliação facial e comportamental da criança ao ouvir as histórias.

Quarta Etapa: os extensionistas foram conhecer e apresentar o projeto de extensão aos coordenadores das instuições, os quais os apresentaram para as equipes de enfermagem. Nesta etapa foi acordada entre as equipes, uma escala mensal, contendo os dias, horários e nomes dos participantes.

Após o acolhimento e capacitação da equipe de extensão, firmou-se o compromisso de reuniões mensais para a organização e avaliação das atividades. Em cada mês, o grupo definia os livros as serem utilizados e escolhiam-se os mais adequados de acordo com cada faixa etária, bem como, os que as crianças mais gostavam segundo a percepção dos contadores. 
Desse modo, em cada reunião, os acadêmicos de enfermagem eram sensibilizados a contar as suas próprias experiências, suas potencialidades e dificuldades, esse diálogo permitia a exposição de ideias e práticas para o aprimoramento do projeto, por meio da escuta, ação e reflexão do grupo (FREIRE P,2019). Contudo, os acadêmicos de enfermagem eram estimulados e preparados para a participação em eventos científicos como a semana de extensão do Programa de Extensão da UFSJ.

\section{DISCUSSÃO}

A internação infantil é um momento difícil na vida da criança e de sua família. A vivência em um ambiente desconhecido causa medo e gera inquietações frente à restrição de suas atividades diárias, sobretudo a sua fragilidade diante da doença, tendo que ser submetida a procedimentos invasivos e dolorosos (GOMES GLL, et.,2016).

As crianças internadas normalmente apresentam-se chorosas e se tornam mais dependentes de seus pais, o que piora o seu estado emocional. As principais repercussões do impacto frente a hospitalização são: o choro, depressão, ansiedade e o medo. Em crianças pequenas podem ocorrer regressões, ou seja, a criança se volta para a fase de desenvolvimento anterior, com o uso de chupetas e fraldas (GOMES GLL, et al.,2016)

A compreensão de que a hospitalização infantil gera repercussões na criança e em sua família, implica para a necessidade de um acolhimento multiprofissional em que se construam mecanismos para atenuar a ansiedade do núcleo familiar. Nesse sentido, incluir a família como unidade de cuidado, confere o entendimento das reações da criança frente à hospitalização em cada faixa etária, para que a equipe, especificamente, a enfermagem possa interagir utilizando-se do lúdico para a execução de suas atividades (AZEVÊDO AVS, et al., 2017).

Diante disso, a vivência do grupo de extensão composta por enfermeiros e graduandos de enfermagem mostrou, segundo as suas percepções, que o lúdico contribui no desenvolvimento e reabilitação da criança, este importante recurso implica em diferentes relações do sujeito com a realidade em que se encontra (OLIVEIRA LE, et al.,2018).

Os autores Nijhof SL, et al. (2018) reafirmam que o comportamento lúdico quando estimulado favorece a adaptabilidade da criança a condição da doença, e esse movimento promove o funcionamento cognitivo, social, emocional e psicomotor, esse processo irá contribuir no futuro para a sua saúde.

Nessa perspectiva, os extensionistas por meio da observação direta e indireta puderam avaliar que crianças debilitadas e impossibilitadas de frequentarem a brinquedoteca, ao ouvir as histórias dos clássicos infantis, e de outros tipos de enredos, mudavam as suas fisionomias, sorriam, alegravam-se e pediam para repetir as histórias. Outro fator positivo desse projeto lúdico foi a contribuição com os voluntários da brinquedoteca hospitalar, visto que a mesma funciona em horários esporádicos, e nestas ocasiões o projeto contos atuava.

Notou-se também que os pais acompanhantes ficavam muito felizes com a presença dos contadores de histórias, e comentavam ser um momento mágico e de volta ao seu passado quando eram crianças. Nesse sentido, ouvir histórias transforma-se em ferramenta de cuidado e humanização também para os acompanhantes, nesse momento eles são capazes de comentar as suas vivências com o paciente e os desafios da hospitalização (SILVA ACM e SEI MB, 2019).

ROCKEMBACH J et al. (2017) revelaram em seu estudo que os pais percebem a importância do lúdico para as crianças, reconhecendo que essa ação muda o foco centrado na doença e hospitalização para uma brincadeira que tranquiliza e ameniza o sofrimento da criança.

Para os profissionais de enfermagem, o projeto contribuiu para a execução de suas intervenções, principalmente no momento da administração de medicamentos em crianças menores, as quais se apresentavam mais temerosas, assim elas se distraiam ao ver as gravuras do livro. FALKE ACS et al. (2018) corroboram com essa questão ao pontuarem em seu estudo que os profissionais demonstraram o reconhecimento do lúdico, como uma forma de cuidado integral, sendo a utilização de brinquedos, brincadeiras e contos infantis, intervenções para um ambiente acolhedor no hospital e um facilitador na interação entre o profissional e a criança. 
De acordo com Silva DO, et al. (2018) o lúdico associado ao jogo, brinquedo, divertimento traz o riso e isso impulsiona a resolução momentânea dos problemas, o que vale é o momento vivido. Nessa perspectiva, o grupo de contadores adotou outras formas de narrar as suas histórias, utilizando-se de fantoches, fantasias e músicas para estimular o imaginário e a comunicação da criança, com o apoio dos pais acompanhantes. Dessa prática foi realizada uma reportagem sobre o projeto que pode ser acessada pelo endereço: projetocontos.ufsj.com no Instagram e Facebook (TV Alterosa, 2019).

$\mathrm{O}$ ato de contar histórias aliado a outros projetos lúdicos, pode favorecer um processo terapêutico, uma vez que partem da perspectiva da humanização do cuidado, melhorando assim a assistência prestada, amenizando o estresse, e aumentando a segurança da criança frente ao ambiente e a equipe multiprofissional, o que promove o seu bem-estar (CARVALHO CBM, et al.,2018; OLIVEIRA OP, et al., 2020).

Embora o enfermeiro possa utilizar o brinquedo terapêutico (BT) como estratégia de intervenção, algumas barreiras são encontradas para a sua utilização em algumas unidades pediátricas, como apontado por Oliveira LE, et al. (2018) no que tange a falta de recursos, estrutura e de aptidão/capacitação para o seu desenvolvimento. Como nas instituições envolvidas não há essa estratégia do BT, o grupo de extensão sentiuse motivado a criar e implementar o projeto Contos, para assim contribuir na interação e no desenvolvimento psíquico das crianças. Para Carvalho CBM, et al., (2018), a ludicidade da história corrobora para a criação de uma zona de desenvolvimento nesses pacientes pediátricos, passível de transformação por meio da aprendizagem, desenvolvendo a imaginação que é um processo psicológico novo para ela.

Acredita-se que a história contada leve os pacientes pediátricos a descoberta de um novo mundo, transportando-os a lugares deslumbrantes saindo mentalmente de seus leitos hospitalares, o que contribiu com o seu desenvolvimento cognitivo e social. É válido ressaltar que o cuidar de crianças em situação de hospitalização, requer dos profissionais a construção de um plano de cuidados que não vise exclusivamente a recuperação física desses pacientes, mas também as reais demandas psicossociais, o que contribui para a humanização e a integralidade da assistência.

\section{CONSIDERAÇÕES FINAIS}

A elaboração e execução desse projeto permitiu aos profissionais de saúde uma reflexão acerca do lúdico na vida de crianças hospitalizadas, como estratégia de cuidado, atenção, comunicação e formação de vínculo. Ademais, a utilização de ferramentas lúdicas apresenta esse potencial de humanizar e garantir o direito da criança de brincar e buscar a manutenção do seu estado emocional.

Embora o presente relato apresente limitações, tendo em vista a sua execução em apenas duas instituições do município, esse projeto lúdico firma o compromisso da universidade em propiciar o ensino, pesquisa e extensão na área de Saúde da Criança e do Adolescente, sensibilizando não somente os acadêmicos do curso de enfermagem, como também a equipe de saúde nas instituições que sediam esta ação. Frente a esse processo dialógico, pactua-se a interdisciplinaridade e interprofissionalidade necessárias a formação do aluno, destacando-o em uma aprendizagem significativa de um projeto com relevância social e humanitária.

\section{REFERÊNCIAS}

1. AZEVEDO AVS, et al. Interação equipe de enfermagem, família, e criança hospitalizada: revisão integrativa. Ciênc. saúde coletiva, 2017; 22(11):3653-3666.

2. BRASIL MS. Política Nacional de Humanização-PNH. Brasília, 2013. Disponível em: https://bvsms.saude.gov.br/bvs/publicacoes/politica_nacional_humanizacao_pnh_folheto.pdf. Acesso em: 18 set. 2020.

3. BRASIL MS. Congresso Nacional. Lei 11.104, de 21 de março de 2005. Dispõe sobre a obrigatoriedade de instalação de brinquedotecas nas unidades de saúde que ofereçam atendimento pediátrico em regime de internação. Diário Oficial da República Federativa do Brasil. Brasília, 2005 mar 21; № 184.

4. CARVALHO CBM. Biblioteca Viva em Hospitais: a importância da leitura como estratégia de humanização, a experiência do instituto Fernandes Figueira. Revista Brasileira de Biblioteconomia e Documentação,2018; 12 (2):143154. 
5. FALKE ACS, et al. Percepción del equipo de enfermería sobre el enfoque lúdico al niño hospitalizado. Cultura de los Cuidados (Edición digital), 2018; 22(50): 12-24.

6. FREIRE P. Pedagogia do oprimido. Rio de Janeiro: Paz e terra, 2013; 256p.

7. GIACOMELLO KJ, MELO LL. The meaning of the care of hospitalized children: experiences of nursing professionals. Rev. Bras. Enferm, 2019; 72(3):251-258.

8. GOMES LL, et al. Hospitalization anxiety in children: conceptual analysis. Rev. Bras. Enferm, 2016; 69(5):940-945.

9. NIJHOF SL, et al. Healthy play, better coping: The importance of play for the development of children in health and disease.Neurosci Biobehav Rev, 2018; 95(1): 421-429.

10. OLIVEIRA LE, et al. Atividades lúdicas desenvolvidas pela enfermagem em um hospital materno infantil. Rev. Ciênc. Ext, 2018; 14(3): 159-169.

11. OLIVEIRA OP, et al. A percepção de crianças escolares acerca da hospitalização: estudo com dados qualitativos. Revista Eletrônica Acervo em Saúde, 2020; 50 (e3409): 1-9.

12. ROCKEMBACH J, et al. Insertion of play as facilitator of hospitalization in childhood: parental perception. .J. nurs. Health,2017; 7(2): 117-126.

13. SILVA ACP, et al. Efeitos da música clássica aplicada em crianças hospitalizadas. Revista Eletrônica Acervo Saúde, 2020; 48:(e3215):6-9.

14. SILVA ACM, et al. A Contação de Histórias e a humanização no hospital: vivências de profissionais da saúde. Rev. SBPH. São Paulo, 2019; 22 (2): 68-89.

15. SILVA DO, et al. The importance of play in the context of child hospitalization la importancia del lúdico en el contexto de la hospitalización infantil. Rev enferm UFPE on line. Recife, 2018; 12(12):3484-3491.

16. TV Alterosa-Centro-Oeste. Conheça o projeto contação de histórias. Divinópolis, 5 junho 2019. Reportagem. Disponível em https://www.youtube.com/watch?v=0EjO52cyfN8\&feature=share. Acesso em: 2 jun.2020. 